\title{
Mature Results of a Prospective Randomized Trial Comparing 5-Flourouracil with Leucovorin to 5-Flourouracil with Levamisole as Ad- juvant Therapy of Stage II and III Colorectal Cancer- The Israel Cooperative Oncology Group (ICOG) Study
}

\section{Arie Figer $^{1}{ }^{*}$, Aviram Nissan ${ }^{*}{ }^{凶}$, Adi Shani ${ }^{3}$, Riva Borovick ${ }^{4}$, Mariana Stiener ${ }^{4}$, Mario Baras ${ }^{5}$, Herbert R. Freund ${ }^{2}$, Aaron Sulkes ${ }^{6}$, Alexander Stojadinovic ${ }^{7}$, Tamar Peretz ${ }^{8}$}

1. Department of Oncology, Tel Aviv-Souraski Medical Center, Tel Aviv, Israel.

2. The Department of Surgery, Hadassah-Hebrew University Medical Center, Jerusalem, Israel.

3. Department of Oncology, Kaplan Medical Center, Rehovot, Israel.

4. Lin Oncology Center, Haifa, Israel.

5. The Department of Biostatistics, Hadassah-Hebrew University Medical Center, Jerusalem, Israel.

6. Department of Oncology, Rabin Medical Center, Petah Tikva, Israel.

7. Department of Surgery, Walter Reed Army Medical Center, and the United States Military Cancer Institute, Washington, D.C., USA.

8. The Department of Oncology, Hadassah-Hebrew University Medical Center, Jerusalem, Israel.

* Share first authorship.

Corresponding author: Aviram Nissan, M.D., Department of Surgery, Hadassah-Hebrew University Medical Center, Mount Scopus, P.O.Box 24035, Jerusalem, Israel. Telephone: +972-2-5845045; Fax: +972-2-5844028; e-Mail: anissan @hadassah.org.il

(C) Ivyspring International Publisher. This is an open-access article distributed under the terms of the Creative Commons License (http://creativecommons.org/ licenses/by-nc-nd/3.0/). Reproduction is permitted for personal, noncommercial use, provided that the article is in whole, unmodified, and properly cited.

Received: 2011.03.10; Accepted: 2011.04.01; Published: 2011.04.01

\begin{abstract}
Objective: Survival benefit with adjuvant therapy was shown in patients with Stage III colorectal cancer (CRC). This study evaluates long-term (I0-year) outcome in patients with CRC randomly assigned to adjuvant 5-Fluorouracil/Leucovorin (5FU+LV) or 5-FU/Levamisole (5FU+LEV).

Methods: Between 1990 and 1995, 398 patients with curatively resected Stage II-III CRC were randomly assigned to adjuvant $5 F U+L V$ or $5 F U+L E V$ for 12 months.

Results: No difference was evident in 10 -year relapse-free or overall survival between study groups. Grade III toxicity was similar between groups; however, neurotoxicity was significantly greater with $5 F U+L E V(p=0.02)$ and gastrointestinal toxicity with $5 F U+L V(p=0.03)$. Female patients treated with 5FU+LEV had improved overall survival.

Conclusions: Adjuvant treatment of CRC is still based on leucovorin modulated fluorouracil. The long-term follow-up results of this trial indicate that the adjuvant treatment of Stage II-III CRC with $5 F U+L V$ or $5 F U+L E V$ is equally effective. The finding of improved survival in female subjects treated with 5FU+LEV warrants further study to determine if Levamisole is a better modulator of 5-FU than Leucovorin in this patient subset.
\end{abstract}

Key words: colorectal cancer, adjuvant chemotherapy, 5-fluorouracil, leucovorin, levamisole, survival 


\section{Introduction}

Colorectal cancer is a major cause of death worldwide $^{1}$. As the risk of disease-recurrence and cancer-specific mortality in patients with tumors invading the bowel wall or regional lymphatics is high, adjuvant therapies have been pursued as a means to improve survival ${ }^{2}$. The first evidence of a survival benefit for adjuvant chemotherapy was reported in the NSABP-C-01 study, the combination of semustine, vincristine and 5-fluorouracil (MOF) was shown to improve 5-year disease-free (DFS) and overall survival (OS) as compared to adjuvant immunotherapy with BCG or no further treatment ${ }^{3}$. However, the DFS and OS benefit associated with adjuvant MOF chemotherapy disappeared after 10 years ${ }^{4}$. An early meta-analysis indicated a small OS benefit with adjuvant non-modulated 5FU indicating the need for further adjuvant therapy trials in AJCC Stage III CRC ${ }^{5}$. The addition of the antihelminthic agent, Levamisole (LEV), to 5FU was first evaluated in the context of a randomized clinical trial by the $\mathrm{NCCTG}^{6}$. In a three-arm clinical trial, the combination of 5FU+LEV administrated for 12 months was found to be superior to either surgery alone or surgery followed by LEV alone. 5-FU+LEV reduced the recurrence rate by $40 \%$ and the death rate by $33 \%$ in Dukes' C colon cancer. A subsequent trial (INT-0035) that randomized patients with Dukes'B2 and C colon cancer to surgery alone, or one year of adjuvant LEV or 5FU+LEV confirmed the efficacy of the 5FU and LEV combination in terms of improved DFS and OS. The results of this trial7-9, established adjuvant therapy for Stage III colon cancer as a standard of care by the National Cancer Institute consensus development panel ${ }^{10}$. The activity of leucovorin (LV)-modulated 5-FU in metastatic colon cancer led to the evaluation of this regimen in the adjuvant setting. The NSABP C-03 trial randomized patients with Dukes' $\mathrm{B}$ and $\mathrm{C}$ to adjuvant bolus 5FU-LV and the MOF combination ${ }^{11}$.

Treatment with LV-modulated 5FU resulted in a significant improvement in outcome over MOF. The International Multicentre Pooled Analysis of Colon Cancer Trials (IMPACT) assessed over 1,400 patients with Stage II/III colon cancer randomized to 6 months of $5 \mathrm{FU}+\mathrm{LV}$ or post-operative observation (IMPACT, 1995). Adjuvant 5FU+LV significantly improved DFS by $35 \%$ and reduced mortality by $22 \%$. A later pooled analysis demonstrated a significant DFS and OS benefit of both LV and LEV-modulated 5FU adjuvant therapy in Stage II/III when compared to surgery alone across all patient subsets ${ }^{12}$. Several groups embarked on clinical trials comparing the two forms of 5FU- based adjuvant therapy ${ }^{12-22}$. A three-arm trial demonstrated that a short 6-month course, dose dense, three-drug regimen of $5 \mathrm{FU}+\mathrm{LV}+\mathrm{LEV}$ was superior to 5FU+LEV given for either 6 or 12 months ${ }^{17}$. NSABP C-04 compared three adjuvant treatment regimens, $5 \mathrm{FU}+\mathrm{LV}$ and $5 \mathrm{FU}+\mathrm{LEV}$ and $5 \mathrm{FU}+\mathrm{LV}+\mathrm{LEV}$, finding similar efficacy with the two drug therapy and no incremental benefit to the three drug combination ${ }^{18}$. The INT $0089^{21}$ and the adjCCA-0119,22 trials compared various dose intensities and durations of $5 \mathrm{FU}+\mathrm{LV}$ to 5FU+LEV and suggested superiority of modulation with Leucovorin over Levamisole, at least for treatment lasting 6 months only. In the majority of these trials the reported follow-up is 5 years. Very few studies report a longer follow up of up to 10 years ${ }^{19}$. We report a long-term follow-up of a study comparing adjuvant $5 \mathrm{FU}+$ Levamisole to $5 \mathrm{FU}+$ Leucovorin for 12 months in Stage II and III CRC. The present clinical trial was designed in 1990 when the initial results of adjuvant clinical trials showing the benefit of $5 F U+L E V$ were reported. The original aim of the study was to compare the two forms of adjuvant therapy evaluating RFS with secondary aims of evaluating $O S$ and toxicity.

The aims of this report are to evaluate the long-term RFS and identify subgroups of patients that may benefit from either form of adjuvant therapy and might modify the current practice modulation of 5FU by Leucovorin in selected patients.

\section{Patients and methods}

The reporting of this clinical trial is in accordance with the CONSORT- consensus statement ${ }^{23}$.

Participants: A national prospective randomized clinical trial was conducted following approval by participating medical center Institutional Review Boards. Between 1990 and 1995, 398 patients with pathological AJCC Stage II-III colorectal cancer underwent complete (R0) resection at four cancer centers in Israel. These patients were then randomly assigned to receive either 5FU+LV $(n=210)$ or 5FU+LEV $(n=188)$ for 12 months. To be eligible patients underwent operation within 6 weeks of study entry, had Karnofsky Performance Status score $>85 \%$, and were over the age of 18 years. Patients with recurrent disease, those who received prior chemotherapy or had prior malignancies, as well as patients with severe co-morbidities, residual or systemic disease were excluded. Patients with primary rectal cancer received adjuvant radiation therapy independent of their group allocation. Patients with rectal cancer who were treated with neaoadjuvant chemoradiation were excluded from the study. Twenty two patients were ineligible for this study (Figure 1). 


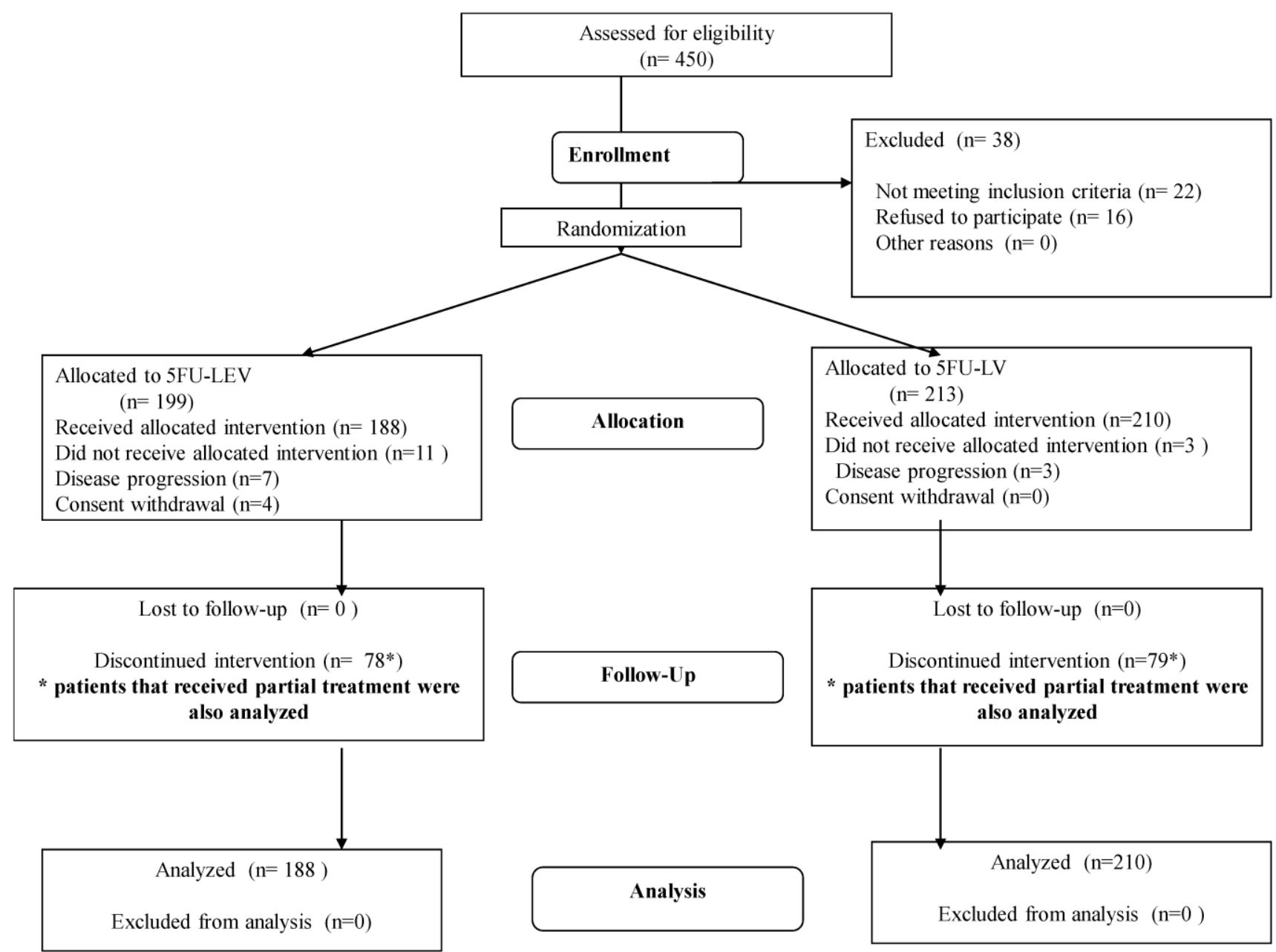

Figure I: Study flow chart.

Interventions: Patients were assigned randomly to receive either adjuvant 5FU+LEV (5FU $450 \mathrm{mg} / \mathrm{m}^{2}$ i.v. bolus daily from days 1 to 5 , then from day 28 , every other week at a dose of $900 \mathrm{mg} / \mathrm{m}^{2} \mathrm{i}$.v. bolus for 12 months + LEV 150mg orally from days 1 to 3 , then from day $28,150 \mathrm{mg}$ orally for three consecutive days every two weeks for 12 months), or adjuvant 5FU+LV (5FU $375 \mathrm{mg} / \mathrm{m}^{2}$ i.v. bolus daily from days 1 to $5+\mathrm{LV}$ $200 \mathrm{mg} / \mathrm{m}^{2}$ i.v. as a 10 minute infusion before $5 \mathrm{FU}$ administration; then from day $28,5 \mathrm{FU} 900 \mathrm{mg} / \mathrm{m}^{2}$ i.v. bolus + LV $100 \mathrm{mg} / \mathrm{m}^{2}$ i.v. over 10 minutes infusion before 5FU administration, every two weeks for 12 months). Toxicity was assessed at each chemotherapy session and scored according to WHO criteria.

Objectives: The principal aim of this trial was to determine if adjuvant 5FU+LV offered a statistically significant improvement in RFS. Secondary endpoints were OS and toxicity over adjuvant 5FU+LEV in the setting of completely resected CRC.

Outcome: Follow-up data was obtained from the four participating institutions. Patients alive without recent follow-up data were contacted and their disease status was updated._The clinical outcomes studied were loco-regional recurrence (LRR), distant recurrence (DR), relapse-free survival (RFS), and overall survival (OS). Time to recurrence, and OS were calculated from the date of surgery. In defining DR, any distant recurrence following definitive surgery was considered.

Sample size: To achieve the probability of a Type I error at alpha $=0.05$, a sample size of 400 subjects per group would have $80 \%$ power to detect a $10 \%$ absolute difference in 5-year RFS between the two treatment arms.

Randomization: Randomization was balanced between the two treatment arms stratified by gender 
(male vs. female), tumor location (colon vs. rectum), grade (low vs. high) and stage. Randomization was carried out with a stratified permuted block scheme using a separate randomization table for each participating study site. The allocation sequence was generated by the study biostatistician.

Statistical methods: The rate of recurrence or death was estimated using the Kaplan-Meier product-limit method and statistical differences in RFS or OS were calculated by the log rank test ${ }^{24}$.

Clinical and pathological covariates investigated in this study included: treatment arm, completion of therapy, age, gender, and ethnicity, disease stage, histological differentiation, tumor location (colon or rectum), family history of cancer, abnormal preoperative CEA, and body mass index (BMI). Clinicopathologic factors were correlated with study endpoints. Associations between categorical factors were studied with Fisher's exact test or Chi-squared test, as appropriate. Toxicity grades were compared with Wilcoxon, Mann-Witney Test for comparison of means. Univariate influence of prognostic factors on study endpoints was analyzed using a stratified log rank test ${ }^{25}$. Multivariate analysis based on Cox's proportional hazards regression model was used to associate covariate to time-dependent endpoints adjusted for major prognostic stratifying variables ${ }^{26}$. The clinical and pathological data were processed using SPSS-10® (SPSS, Chicago IL). A p value $<0.05$ was considered statistically significant. All P values are two sided.

\section{Results}

\section{Patient characteristics}

Between 1990 and 1995, 398 patients were randomly assigned to receive either 5FU+LEV $(\mathrm{N}=188)$ or 5FU+LV ( $\mathrm{N}=210)$ (Figure 1, Study Flow). Baseline demographic data are reported in Table $\mathbf{1}$ demonstrating no significant imbalance between the two treatment groups. Median age was 63 years (range 24-76 years) with a slight male to female predominance. The majority of patients in the study were treated for adenocarcinoma of the colon $(81 \%$; rectum $19 \%)$. Two hundred forty-one $(60.6 \%)$ patients successfully completed 12 months of either treatment protocol. There was no major difference in number of patients completing adjuvant therapy between the $5 F U+L E V$ group $(110 / 188,58.5 \%)$ and the 5FU+LV group $(131 / 210,62.4 \%, \mathrm{p}=0.15)$.

Treatment was not completed according to the study protocol in 157 patients $(39.4 \%)$ mainly due to toxicity $(13.4 \%)$, disease recurrence $(9.5 \%)$ or protocol violation $(16.3 \%)$.
Table I: Patient and tumor characteristics.

\begin{tabular}{|c|c|c|c|c|}
\hline & $\begin{array}{l}\text { All } \\
\text { patients } \\
(\mathrm{n}=398)\end{array}$ & $\begin{array}{l}\text { 5FU-LEV } \\
(\mathrm{n}=188)\end{array}$ & $\begin{array}{l}5 \text { FU-LV } \\
(n=210)\end{array}$ & $\mathrm{p}_{\text {Value }}$ \\
\hline Median age & 63.0 & 61.8 & 61.3 & 0.31 \\
\hline Minimum & 24.0 & & & \\
\hline Maximum & 76.0 & & & \\
\hline Gender & & & & 0.66 \\
\hline Male & 210 & $97(52 \%)$ & $113(54 \%)$ & \\
\hline Female & 188 & $91(48 \%)$ & $97(46 \%)$ & \\
\hline Ethnicity & & & & 0.39 \\
\hline Ashkenazi & 252 & $112(60 \%)$ & $140(67 \%)$ & \\
\hline Non-Ashkenazi & 131 & $67(36 \%)$ & $64(30 \%)$ & \\
\hline Non-Jewish & 15 & $9(4 \%)$ & $6(3 \%)$ & \\
\hline $\begin{array}{l}\text { Anatomic loca- } \\
\text { tion }\end{array}$ & & & & 0.63 \\
\hline Colon & 322 & $154(82 \%)$ & $168(80 \%)$ & \\
\hline Rectum & 76 & $34(18 \%)$ & $42(20 \%)$ & \\
\hline AJCC Stage & & & & 1.00 \\
\hline Stage II & 197 & $93(50 \%)$ & $104(50 \%)$ & \\
\hline Stage III & 201 & $95(50 \%)$ & $106(50 \%)$ & \\
\hline $\begin{array}{l}\text { Histological } \\
\text { grade (differen- } \\
\text { tiation) }\end{array}$ & & & & 0.22 \\
\hline Well & 100 & $49(26 \%)$ & $51(24 \%)$ & \\
\hline Moderately & 210 & $105(56 \%)$ & $105(50 \%)$ & \\
\hline Poor & 44 & $16(9 \%)$ & $28(13 \%)$ & \\
\hline Not-recorded & & $17(9 \%)$ & $27(13 \%)$ & \\
\hline \multicolumn{5}{|l|}{$\begin{array}{l}\text { Performance } \\
\text { status }\end{array}$} \\
\hline 0 & 351 & $\begin{array}{l}169 \\
(48.2 \%)\end{array}$ & $182(51.8 \%)$ & \\
\hline 1 & 44 & $20(45.5 \%)$ & $24(54.5 \%)$ & \\
\hline 2 & 3 & $1(33.3)$ & $2(66.7 \%)$ & \\
\hline
\end{tabular}

\section{Toxicity}

The vast majority of the toxic events in both groups occurred between the $4^{\text {th }}$ and the $9^{\text {th }}$ month of therapy. Although the absolute number of toxic events were similar in both treatment groups, events of treatment discontinuation occurred earlier in the $5 F U+L E V$ group $(p=0.02)$. Adverse effects related to adjuvant systemic therapy differed significantly between the two treatment arms (Table 2). Increased frequency of Grade III-IV neurotoxicity was observed in the 5FU+LEV group $(p=0.02)$ while Grade III-IV gastrointestinal toxicity predominated in the $5 \mathrm{FU}+\mathrm{LV}$ group ( $p=0.03$ ). Hematological toxicity was mild and no episode of febrile neutropenia occurred. There was no death potentially attributable to chemotherapeutic treatment. Despite the fact that there was no difference in the relative portion of patients who failed to complete the 12 -month treatment protocol, only $71.7 \%$ of 5FU+LEV-treated patients received treatment for 
more than 6 months, compared to $80.9 \%$ of the $5 F U+L V$ group, $p=0.04$. Age, gender, and ethnicity, disease stage, tumor location (colon or rectum), and body mass index (BMI) had no association with toxicity.

Table 2: Grade 3-4 toxicity profile of the 2 study arms.

\begin{tabular}{llll}
\hline & $\begin{array}{l}5 \mathrm{FU}-\mathrm{LEV} \\
(\mathrm{n}=188)\end{array}$ & $\begin{array}{c}5 \mathrm{FU}-\mathrm{LV} \\
(\mathrm{n}=210)\end{array}$ & $\mathrm{p}$ Value \\
\hline $\begin{array}{l}\text { Hematological } \\
\text { grade 3-4 }\end{array}$ & & & \\
Anemia & $2.0 \%$ & $2.0 \%$ & 0.67 \\
$\quad$ Neutropenia & $8.0 \%$ & $14 \%$ & 0.09 \\
$\quad$ Thrombocytopenia & $3.0 \%$ & $4.0 \%$ & 0.32 \\
$\begin{array}{c}\text { Neurological grade 3-4 } \\
\text { Ataxia }\end{array}$ & $9.6 \%$ & $3.8 \%$ & 0.02 \\
$\quad$ Dizziness & $28.7 \%$ & $18.6 \%$ & 0.05 \\
Gastrointestinal grade 3-4 & & & \\
$\quad$ Diarrhea & $0.0 \%$ & $2.0 \%$ & 0.03 \\
$\quad$ Nausea & $0.0 \%$ & $3.0 \%$ & 0.02 \\
Muco-cutaneous grade 3-4 & & & \\
$\quad$ Stomatitis & $0.0 \%$ & $1.0 \%$ & 0.74 \\
$\quad$ Alopecia (partial) & $0.0 \%$ & $0.0 \%$ & 0.26 \\
\hline
\end{tabular}

\section{Disease outcome: Relapse-free Survival}

Actuarial (Kaplan-Meier) survival analysis was performed in January 2004 with a median overall follow up time of 99 months for the entire group, and 138 months for the patients alive at time of last contact. Ten patients $(2.5 \%)$ were lost to follow-up. Recurrence data was unavailable for 13 patients, leaving 385 patients for relapse-free analysis. There were 122 relapse events in the entire study group, 54 in the 5FU+LEV and 68 in the $5 F U+L V$ group $(p=0.59)$.

Distribution of recurrence (35 local-regional and 87 distant) by anatomic location for the entire study group is summarized in Table 3 . There were 16 $(13.1 \%)$ delayed (>5years) recurrence events, 9 in the $5 F U+L E V$ and 7 in the 5FU+LV group. The latest recurrence occurred 113 months after randomization.
Five- and ten-year RFS rates for the 5FU+LEV and $5 \mathrm{FU}+\mathrm{LV}$ groups were similar, $72 \%$ and $61 \%$ versus $67 \%$ and $60 \%(p=0.88)$, respectively, and were not influenced by any clinical variable recorded.

Table 3: Relapse by location.

\begin{tabular}{ll}
\hline Locoregional: & 35 \\
\hline Distant: & 46 \\
Hepatic & 22 \\
Pulmonary & 5 \\
Peritoneal & 1 \\
Brain & 13 \\
Unspecified distant recurrence & 122 \\
\hline Total & \\
\hline
\end{tabular}

\section{Disease outcome: Overall Survival}

Survival status at the time of analysis was available for all study patients $(\mathrm{n}=398) ; 156$ died $(5 \mathrm{FU}+\mathrm{LEV}, \mathrm{n}=74$ vs. $5 \mathrm{FU}+\mathrm{LV}, \mathrm{n}=82)$. Five- and ten-year OS rates for the 5FU+LEV and 5FU+LV groups were similar, $75 \%$ and $53 \%$ versus $72 \%$ and $59 \%$, respectively, and were not significantly different when analysis was confined only to those patients completing treatment $(\mathrm{p}=0.64)$.

Exploratory sub-group analysis found significantly better OS patients who completed more than 6 months of therapy versus those that did not, overall $(\mathrm{p}=0.002)$ and in each study group $(5 \mathrm{FU}+\mathrm{LEV}, \mathrm{p}=$ 0.046 ; $5 \mathrm{FU}+\mathrm{LV}, \mathrm{p}=0.02$ : Figure $2 \mathrm{~A}, \mathbf{B}, \mathbf{C})$.

Gender-specific subset analysis identified a trend toward improved RFS and statistically significant improved OS for female patients (10-year OS: M vs. $F=53 \%$ vs. $64 \% ; p=0.04$, Figure $3 a$ ). This difference was evident only in the 5FU+LEV group (5FU+LEV: 10-year OS: M vs. F $=46 \%$ vs. $71 \%$; $p=0.04$, Figure $3 \mathbf{b}$ ).

On a Cox stepwise regression multivariate analysis only AJCC Stage III emerged as an independent predictor of relapse-free and overall survival (RFS: $\mathrm{RR}=3.43$ [95\% CI 2.08-5.66], $\mathrm{p}=0.0001 ; \mathrm{OS}: \mathrm{RR}=1.5$ [1.3-1.8], $\mathrm{p}=0.001)$. 

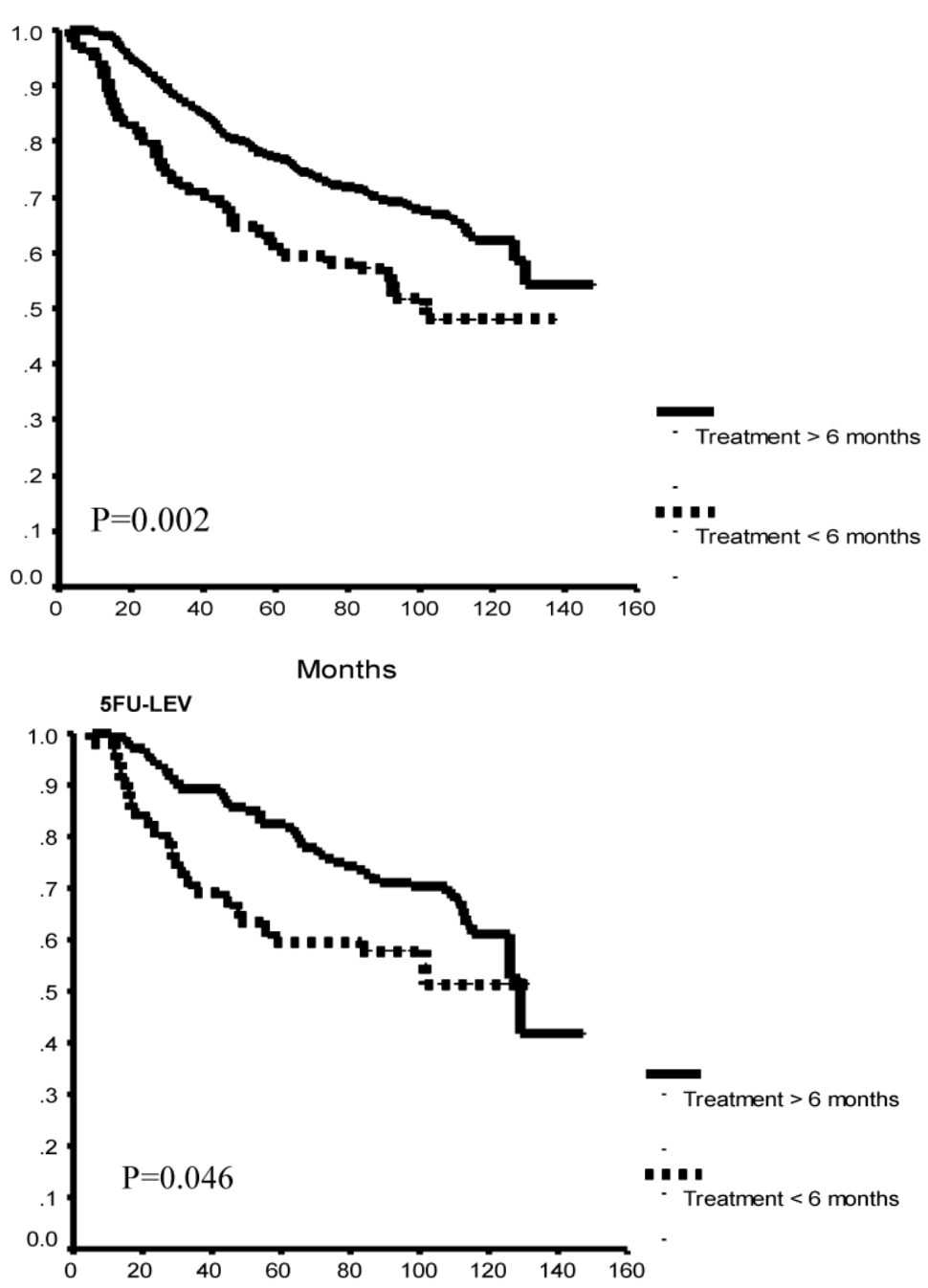

Months

5FU-LV

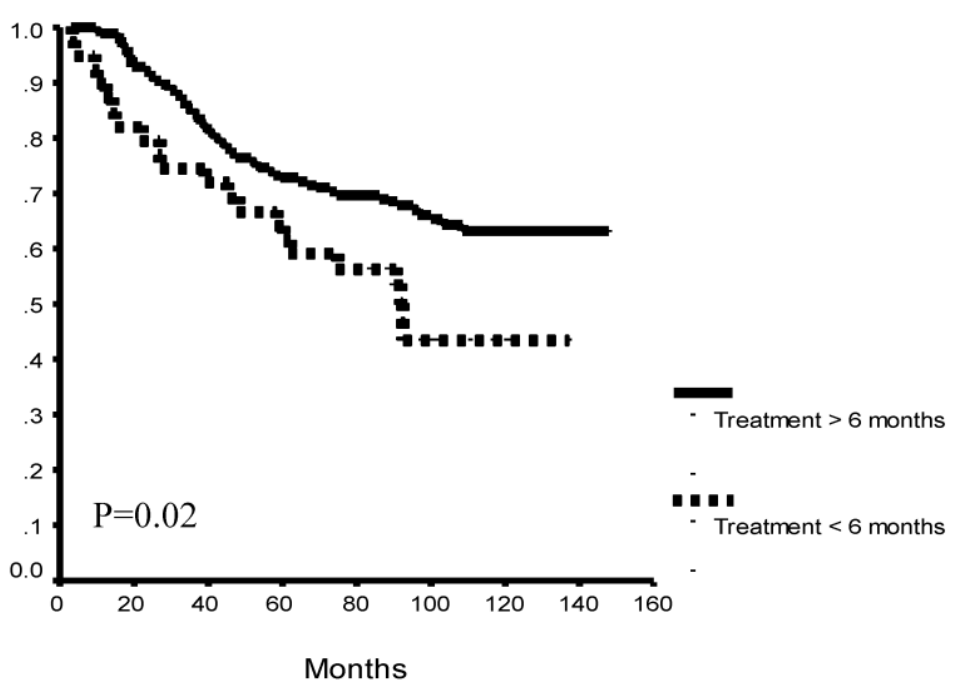

Figure 2: Overall survival as a function of protocol completion. Patients who completed 12 months of either treatment protocol had significantly better overall survival as compared to patients who discontinued their treatment before completion of at least 6 months of therapy. This survival difference was seen when all study patients $(\mathbf{A})$ and for each of the study arms (B, C). 


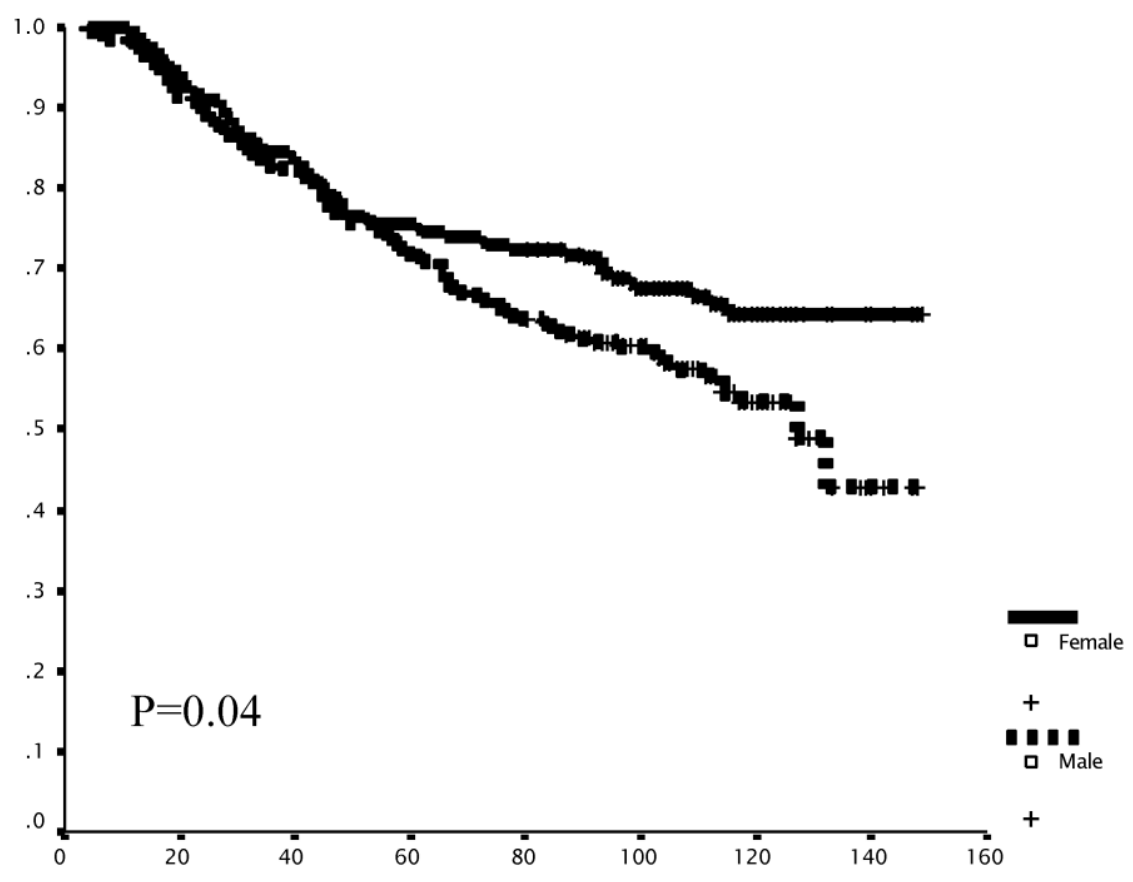

Months

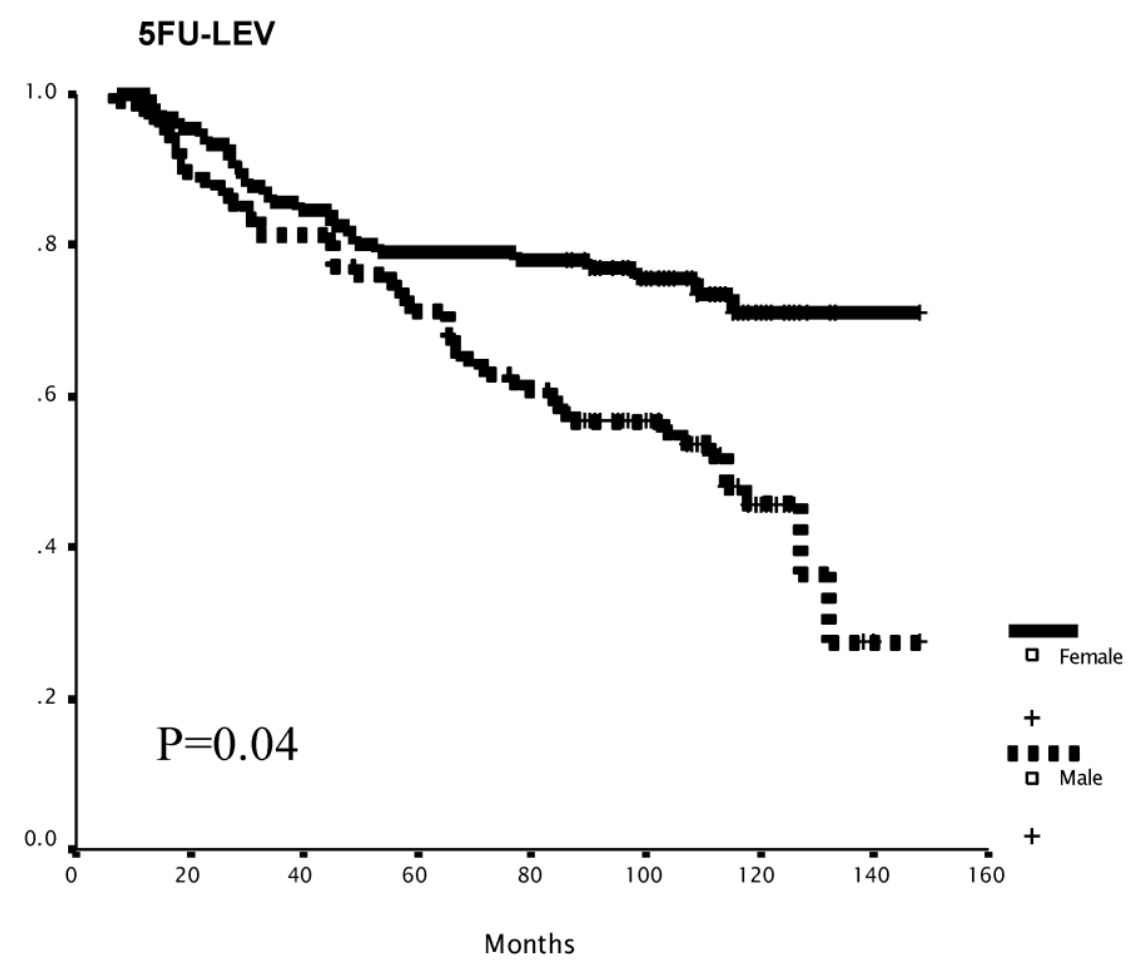

Figure 3: Survival as a function of gender. The overall survival of female patients was significantly better than that of male patients (A). Mean survival was 102.4 (95\% C.I 95.I-109.7) and II 3.7 (95\% C.I 106.3-12I.4) months for the male and the female groups respectively, log - rank $p=0.04$. In the $5 F U+L E V$ group $(n=188)$. The overall survival of female patients $(n=91)$ was significantly better than the OS of male patients $(n=97)$ in the 5FU+LEV group (B). Mean survival was $96.3 .4(95 \%$ C.I 86.0-106.6) and I I 9.9 (95\% C.I I09.9- I 29.8) months for the male and the female groups respectively, log -rank $p=0.04$. In the $5 F U+L V$ group $(n=2 \mid 10)$. The overall survival of female patients $(n=97)$ was no different than the OS of male patients $(n=1 \mid 3)$ in the 5FU+LV group. Mean survival was 107.8 (95\% C.I 98.3-I I 7.4) and I07.2 (95\% C.I 96.6-I I7.8) months for the male and the female groups respectively, log -rank $p=0.10$. 


\section{Discussion}

This ICOG trial was designed at a time when Levamisole-modulated 5-FU was standard adjuvant chemotherapy for node-positive (Stage III) CRC. The principal aim of the study was to evaluate RFS with secondary aims of OS as well as toxicity evaluation in patients with Stage II/III CRC randomized to receive 12 months of Leucovorin (5FU+LV)- or Levamisole (5FU+LEV)-modulated 5FU following potentially curative resection. Both LV- and LEV- modulated 5FU in this study were well tolerated with no treatment-related mortality. Ten-year RFS and OS were similar between treatment groups, as was Grade III toxicity; however, neurotoxicity was significantly more likely with 5FU+LEV, and gastrointestinal toxicity with $5 \mathrm{FU}+\mathrm{LV}$. Female patients had significantly improved OS over male patients (10-year, $64 \%$ v. $53 \%$, $\mathrm{p}=0.04$ ), a benefit observed only in the 5FU+LEV treated arm.

The standard of care in adjuvant therapy for patients with resected AJCC Stage III colon cancer is 6 months of $5 \mathrm{FU}+\mathrm{LV}$ with an estimated $33 \%$ reduction in the risk of disease recurrence ${ }^{27}$. Current practice guidelines for adjuvant treatment of Stage II CRC remain controversial ${ }^{28}$. Adjuvant $5 \mathrm{FU}+\mathrm{LV}$ and 5FU+LEV were compared in three clinical trials ${ }^{17,18,21}$. In the Intergroup 0089 trial, there was no significant difference in RFS or OS between the 5 treatment arms. The NSABP CO- 4 trial demonstrated improved RFS and OS for patients treated with 6 months of adjuvant $5 F U+L V$ compared to 12 months of 5FU+LEV. The NCCTG 894651 trial showed that a three-drug regimen, 5-FU/LEV/LV for 6 months resulted in longer OS than 6 months of adjuvant 5-FU+LEV. However, 5-FU+LEV for 12 months were shown to be as effective as the three-drug regimen.

The results of our ICOG trial showed no difference in either 5-year or 10-year RFS or OS between $5 \mathrm{FU}+\mathrm{LEV}$ and $5 \mathrm{FU}+\mathrm{LV}$; however, this trial did not have sufficient statistical power to detect small differences in outcome between the treatment arms, and it was not designed with sufficient power to determine treatment effect prospectively in specific patient subsets.

Present-day novel agents were not used routinely as salvage therapy during the course of this study. In our study, the 5-year OS rates were $85 \%$ and $63 \%$ for Stage II and III CRC, respectively, which are comparable to previously published reports ${ }^{20}$. Also, there was a trend towards improved RFS and significantly improved OS in female patients. Although in larger studies ${ }^{11,19}$ gender did not appear to influence outcome, in a retrospective analysis of 5-FU+LEV 12-month ${ }^{29}$ survival benefit was observed in female patients with right-sided microsatellite unstable tumors. The hypothesis-generating finding of improved long-term OS and a trend towards improved RFS in female patients treated with 5FU+LEV as compared to $5 \mathrm{FU}+\mathrm{LV}$ in the current ICOG trial was not reported previously in prospective adjuvant trials. If this preliminary observation is verified in meta-analysis of randomized clinical trials, then female patient sub-groups may indeed benefit from modulation of $5 F U$ by Levamisole in the context of modern systemic therapy regimens.

The toxicity profile differed somewhat between $5 \mathrm{FU}+\mathrm{LEV}$ and 5FU+LV treatment groups. These findings are consistent with those of others ${ }^{21}$; however, it is important to note that patients in the 5FU+LEV group showed a tendency to earlier discontinuation of adjuvant therapy due to treatment-related toxicity. Despite the introduction of novel therapeutic agents for CRC, LV-modulated 5FU remains an essential part of existing multi-agent adjuvant treatment protocols ${ }^{30}$.

Since the completion of the current study, the addition of Oxaliplatin to 5FU+LV evaluated in the MOSAIC trial ${ }^{31}$ was found to prolong event-free survival relative to $5 \mathrm{FU}+\mathrm{LV}$ alone in Stage III CRC.

Despite advances in terms of progression-free survival for targeted therapy in advanced (Stage IV) CRC, LV-modulated 5FU with or without Oxaliplatin remains a modern-day standard adjuvant therapy in non-metastatic, node-positive CRC. Considering the findings of the current ICOG Trial, 5FU+LEV may remain a viable option in selected groups of CRC patients. The fact that $13 \%$ of CRC recurrences developed after $>5$ years of follow-up, provides key insight into the natural history of the disease and further underscores the importance of long-term follow-up of patients with CRC.

\section{Acknowledgements}

We would like to thank Mrs. Tamar Hamburger for excellent work as database manager and study coordinator.

This work was supported in part by the Israel Cancer Association (special grant).

\section{Conflict of Interest}

The authors have declared that no conflict of interest exists.

\section{References}

1. Jemal A, Murray T, Samuels A, Ghafoor A, Ward E, Thun MJ. Cancer Statistics, 2003. CA Cancer J Clin 2003;53:5-26 
2. Eisenberg B, Decosse JJ, Harford F, Michalek J. Carcinoma of the colon and rectum: The natural history reviewed in 1704 patients. Cancer 1982:49:1131-1134

3. Wolmark N, Fisher B, Rockette H, et al. Postoperative adjuvant chemotherapy or BCG for colon cancer: results from NSABP protocol C-01. J Natl Cancer Inst 1988;80: 30-36

4. Smith RE, Colangelo L, Wieand HS, Begovic M, Wolmark N. Randomized trial of adjuvant therapy in colon carcinoma: 10-year results of NSABP protocol C-01. J Natl Cancer Inst 2004;96:1128-1132

5. Buyse M, Zeleniuch-Jacquotte A, Chalmers TC. Adjuvant therapy of colorectal cancer: Why we still don't know. JAMA 1988;259:3571-3578.

6. Laurie JA, Moertel CG, Fleming TR, Wieand HS, Leigh JE, Rubin J, McCormack GW, Gerstner JB, Krook JE, Malliard J, et al. Surgical adjuvant therapy of large-bowel carcinoma: An evaluation of levamisole and the combination of levamisole and fluorouracil: The North Central Cancer Treatment Group and the Mayo Clinic. J Clin Oncol 1989;7:1447-1456

7. Moertel CG, Fleming TR, Macdonald JS, Haller DG, Laurie JA, Tangen CM, Ungerleider JS, Emerson WA, Tormey DC, Glick $\mathrm{JH}$, et al. Intergroup study of fluorouracil plus levamisole as adjuvant therapy for stage II/Dukes' B2 colon cancer. J Clin Oncol 1995; 13:2936-2943

8. Moertel CG, Fleming TR, Macdonald JS, Haller DG, Laurie JA, Goodman PJ, Ungerleider JS, Emerson WA, Tormey DC, Glick $\mathrm{JH}$, et al. Levamisole and fluorouracil for adjuvant therapy of resected colon carcinoma. N Engl J Med; 1990;322:352-358

9. Moertel CG, Fleming TR, Macdonald JS, Haller DG, Laurie JA, Tangen CM, Ungerleider JS, Emerson WA, Tormey DC, Glick $\mathrm{JH}$, Veeder MH, Mailliard JA. Fluorouracil plus levamisole as effective adjuvant therapy after resection of stage III colon carcinoma: A final report. Ann Intern Med 1995; 122:321-326

10. NIH Consensus Conference: Adjuvant therapy for patients with colon and rectal cancer. JAMA 1990;264:1444-1450.

11. Wolmark N, Rockette H, Fisher B, et al. The benefit of leucovorin-modulated fluorouracil as postoperative adjuvant therapy for primary colon cancer: Results from National Surgical Adjuvant Breast and Bowel Project Protocol C0-3. J Clin Oncol 1993;11:1879-1887

12. Gill S, Loprinzi CL, Sargent DJ, Thomé SD, Alberts SR, Haller DG, Benedetti J, Francini G, Shepherd LE, Francois Seitz J, Labianca R, Chen W, Cha SS, Heldebrant MP, Goldberg RM. Pooled analysis of Fluorouracil-based adjuvant therapy for stage II and III colon cancer: who benefits and by how much? J Clin Oncol 2004;22:1797-1806

13. International Multicenter Pooled Analysis of Colon Cancer Trials (IMPACT): Efficacy of adjuvant fluorouracil and folinic acid in colon cancer. Lancet; 1995;345:939-944

14. O'Connell MJ, Mailliard JA, Kahn MJ, Macdonald JS, Haller DG, Mayer RJ, Wieand HS. Controlled trial of fluorouracil and low-dose leucovorin given for 6 months as postoperative adjuvant therapy for colon cancer. J Clin Oncol 1997;15:246-250

15. Francini G, Petrioli R, Lorenzini L, Mancini S, Armenio S, Tanzini G, Marsili S, Aquino A, Marzocca G, Civitelli S, et al. Folinic acid and 5-fluorouracil as adjuvant chemotherapy in colon cancer. Gastroenterology 1994;106:899-906

16. Shoup MC, Nissan A, Dangelica MI, Tschmelitsch J. Randomized clinical trials in colon cancer. Surg Oncol Clin N Am 2002;11:133-148

17. Wolmark N, Rockette H, Mamounas E, et al. Clinical trial to assess the relative efficacy of fluorouracil and leucovorin, fluorouracil and levamisole, and fluorouracil, leucovorin, and levamisole in patients with Dukes' B and C carcinoma of the colon: results from National Surgical Adjuvant Breast and Bowel Project C-04. J Clin Onco 1999;11: 3553-3559
18. O'Connell MJ, Laurie JA, Kahn M, Fitzgibbons RJ Jr, Erlichman C, Shepherd L, Moertel CG, Kocha WI, Pazdur R, Wieand HS, Rubin J, Vukov AM, Donohue JH, Krook JE, Figueredo A. Prospectively randomized trial of postoperative adjuvant chemotherapy in patients with high-risk colon cancer. J Clin Oncol 1998;1: 295-300

19. Arkenau HT, Bermann A, Rettig K, Strohmeyer G, Porschen R; Arbeitsgemeinschaft Gastrointestinale Onkologie. 5-Fluorouracil plus leucovorin is an effective adjuvant chemotherapy in curatively resected stage III colon cancer: long-term follow-up results of the adjCCA-01 trial. Ann Oncol 2003;3: 395-399

20. Mamounas E, Wieand S, Wolmark N, Bear HD, Atkins JN, Song $\mathrm{K}$, Jones J, Rockette H. Comparative efficacy of adjuvant chemotherapy in patients with Dukes' B versus Dukes' C colon cancer: results from four National Surgical Adjuvant Breast and Bowel Project adjuvant studies (C-01, C-03 and C-04). J Clin Oncol 1999;5:1349-1355

21. Haller DG, Catalano PJ, Macdonald JS, Frontiera MS, Jackson DV, Mayer RJ. Phase III study of fluorouracil, leucovorin, and levamisole in high-risk stage II and III colon cancer: final report of Intergroup 0089. J Clin Oncol 2005;23:8671-8678

22. Porschen R, Bermann A, Löffler T, Haack G, Rettig K, Anger Y, Strohmeyer G; Arbeitsgemeinschaft Gastrointestinale Onkologie. Fluorouracil plus leucovorin as effective adjuvant chemotherapy in curatively resected stage III colon cancer: results of the trial adjCCA-01. J Clin Oncol 2001;19:1787-1794

23. Moher D, Schulz KF, Altman DG. CONSORT. The CONSORT statement: revised recommendations for improving the quality of reports of parallel group randomized trials. BMC Med Res Methodol 2001;1:2

24. Kaplan EL, Meier P. Nonparametric estimation from incomplete observations. Journal of the American Statistical Association 1958;53:457-481

25. Peto R, Peto J. Asymptotically efficient rank invariant procedures. J R Stat Soc (A) 1972;135:185-207

26. Cox DR. Regression models and life-tables. J R Stat Soc (B) 1972;34:187-220

27. Macdonald JS. Adjuvant therapy for colon cancer. CA Cancer J Clin 1999:49: 202-219

28. Benson AB 3rd, Schrag D, Somerfield MR, Cohen AM, Figueredo AT, Flynn PJ, Krzyzanowska MK, Maroun J, McAllister P, Van Cutsem E, Brouwers M, Charette M, Haller DG. American Society of Clinical Oncology Recommendations on Adjuvant Chemotherapy for Stage II Colon Cancer. J Clin Oncol 2004;22: 3408 - 3419

29. Elsaleh H, Joseph D, Grieu F, Zeps N, Spry N, Iacopetta B. Association of tumour site and sex with survival benefit from adjuvant chemotherapy in colorectal cancer. Lancet 2000;355(9217):1745-1750

30. Ychou M, Raoul J, Douillard J, Bugat R, Mineur L, Viret F, Becouarn $Y$, Bouche $\mathrm{O}$, Jacob J, et al. A phase III randomized trial of LV5FU2+CPT-11 vs LV5FU2 alone in adjuvant high risk colon cancer (FNCLCC Accord02/FFCD9802). Proceeding of ASCO; 2005;: 3502a.

31. André T, Boni C, Mounedji-Boudiaf L, Navarro M, Tabernero J, Hickish T, Topham C, Zaninelli M, Clingan P, Bridgewater J, Tabah-Fisch I, de Gramont A, et al. Oxaliplatin, fluorouracil, and leucovorin as adjuvant treatment for colon cancer. N Engl J Med. 2004;350(23):2343-2351 